\title{
Stress relief: emerging methods to mitigate dissociation-induced artefacts
}

Léo Machado $^{1}$, Frederic Relaix ${ }^{1,2,3,4^{*}}$, and Philippos Mourikis ${ }^{1 *}$.

1. Univ Paris Est Créteil, INSERM, IMRB, F-94010 Créteil, France

2. EnvA, IMRB, F-94700 Maisons-Alfort, France

3. EFS, IMRB, F-94010 Creteil, France

4. AP-HP, Hopital Mondor, Service d'histologie, F-94010 Creteil, France

* Corresponding authors:

philippos.mourikis@inserm.fr

http://mourikis-group.webnode.com/

$\&$

frederic.relaix@inserm.fr

https://www.relaixlab.com/

Twitter@FRelaix

Keywords: Stress response, cell dissociation artefacts, single-cell reference atlases, early-response genes, dissociation-induced genes. 


\section{Glossary}

-dissociation-induced artefacts: modifications of the molecular profile of a cell induced during its enzymatic and mechanical dissociation. Most often, these refer to transcriptional changes since they are the most extensively studied, yet histone modifications, like H3K27ac, and post-translational protein modifications have also been reported.

-single-nucleus RNA-seq: RNA sequencing method for profiling gene expression in extracted nuclei. This method relies on direct extraction of nuclei from tissue and does not involve a cell dissociation step. In addition, it allows to recover and analyse nuclei from syncytia, like the myofibres, which are not accessible by standard cell isolation techniques.

-early-response genes: a group of genes that are rapidly induced, usually within minutes, in response to a variety of environmental stressors. The earliest known and best characterized include fos, $m y c$ and jun, amongst others.

-psychrophilic proteases: also known as cryophilic, are proteases that demonstrate enzymatic activity at temperatures as low as $6^{\circ} \mathrm{C}$. These cold-active proteases derive from organisms thriving at near-zero temperatures, like the soil bacterium Bacillus licheniformis from Himalayan glaciers.

-Thiouracil (TU)-tagging: a method for covalently labelling newly transcribed RNA in vivo. Tagging is achieved by the uracil phospho-ribosyltransferase (UPRT) enzyme together with 4thiouracil (4TU), which is acts as a substate. The thio-RNA is biotinylated and streptavidin beads can be used for purification from complex tissues. Transgenic animal models expressing inducible UPRT have been developed, which allow isolation of de novo transcribed RNA from specific cells.

-in situ Fixation (iSiFi): a method to capture the molecular profile of cells as they exist in their native tissue context. This method is based on paraformaldehyde fixation of cells in the tissue prior to the mechanical/enzymatic dissociation. The recovered cells have been shown to be compatible with RNA-seq, ChIP-seq and reduced representation bisulfite sequencing (RRBS) for DNA methylation. 


\begin{abstract}
The rapid progress of single-cell RNA-sequencing at large scales has led to what seemed impossible until recently, the generation of comprehensive transcriptional maps of nearly all cells in multicellular tissues. We pinpoint three key elements as critical to the production of these maps: scalability, spatial information, and accuracy of the transcriptome of the individual cells. Here, we discuss the ramifications of traditional cell-isolation protocols when capturing the transcriptional signature of cells as they exist in their native tissue context, the methods that have been developed to avoid these distortions, and the biological processes that have unravelled on account of these upgraded methodological approaches.
\end{abstract}

\title{
Impact of isolation techniques on the molecular profile of a cell
}

Since the first description of a cell [1] and the formulation of its theory [2], many cellular attributes have been employed to define cell types in multicellular organisms such as shape, anatomical location and function. With the emergence of biology's central dogma [3], the macro-molecular content of a cell, such as its RNA or protein composition, became preponderantly used for cellular ontology segregation. Among them, the transcriptomic profile of a cell has received the most attention from modern cellular biologists. Despite the variable RNA to protein correlations [4], the transcriptome of a cell is a very convenient proxy of its proteome and has been studied extensively, notably via singlecell RNA-sequencing (scRNA-seq), to infer lineage identity, active biochemical pathways and cellular functions. Such inferences have allowed researchers to match physiological and pathological states with the activity of specific genes and pathways as well as segregating cells in remarkably elaborate subgroups. The amount of high-throughput transcriptional maps published is expanding at a restless pace and recently, large scale projects, such as the Human Cell Atlas or murine cellular atlases, undertook the daunting task of systematically sequencing all cells from mammalian organisms [5-7].

Every cell in a multicellular organism is in constant interaction with its local microenvironment that contains both cellular and non-cellular components, including secreted growth factors, the extracellular matrix, circulating hormones, nutrients and oxygen. Cell isolation protocols drastically disrupt the cellular microenvironment and are based on a combination of mechanical dissociation and lengthy enzymatic treatments, often at $37^{\circ} \mathrm{C}$. In light of these facts, the hypothesis that the mere process of isolating a cell from its niche significantly alters its gene expression profile appears reasonable.

One of the first reports on how sample preparation could alter transcriptomic data came from an early microarray-based study, reporting that human peripheral blood cells stored at room temperature overnight induced the expression of thousands of genes, including early-response genes [8]. A decade later, reproducibility difficulties between laboratories in fluorescence-activated cell sorting (FACS) profiles and efficiency of organoid formation from human breast cell preparations were reported [9]. After carefully comparing protocols, the authors found that length and agitation frequency of the proteolytic enzyme collagenase during the digestion step explained the experimental discrepancies. This finding was confirmed at the transcriptional level by the induction of stress-related genes in dissociated neurons [10] and later in muscle stem cells (MuSCs) [11-13] and kidney cells [14]. Since then, virtually all cell-types have been shown to express a characteristic stress signature given sufficient dissociation time. 


\section{Emerging methods for capturing transcriptomes as they exist in their native tissue context.}

Several groups across the world undertook in parallel the task of circumventing dissociation-induced artefacts in high-throughput studies. These efforts resulted in a handful of emerging methods, each with their own strengths and weaknesses. Such methods can help elucidate the impact of dissociation and the prevalence of cellular stress across a broad range of biological conditions, organisms and cell types (Table 1).

\section{Direct extraction of nuclei from tissues for single-nucleus RNA-seq}

It goes without saying that an obvious strategy to avoid the impact of dissociation is to exclude it from the experimental protocol. In that spirit, single-nucleus RNA-seq (snRNA-seq), which relies on direct lysis of intact tissues for extraction and barcoding of single nuclei [15-17], proposes several advantages over other methods for large-scale projects [18].

A seminal study by B. Lacar and colleagues demonstrated that hippocampal neurons isolated by standard procedures were highly expressive in early response genes Jun, Arc and Fos [10], a puzzling result considering that products of these genes were almost undetectable by immunofluorescence on resting hippocampal neurons in vivo. This prompted the authors to use snRNA-seq and demonstrate that the presence of the early response genes was in fact an artefact of the dissociation procedure and that unstimulated neurons do not express them. This discovery was confirmed by snRNA-seq in the mouse visual cortex [19], kidney [20, 21], and in the liver and skeletal muscle [22].

Of importance, it has been shown that the nuclear transcriptome largely reflects its whole-cell counterpart [18]. However, some discrepancies are observed, including the identification of more intronic sequences from unsliced pre-mRNA, which might be reflective of the de novo transcript production, and the failure to capture cytosolic RNAs, including miRNAs and lncRNAs. Moreover, in the context of skeletal muscle and liver, snRNA-seq detected the expected cellular populations, albeit with different relative abundances compared to RNA-seq on dissociated cells. Specifically, we found an under-representation of endothelial cells in snRNA-seq datasets from intact tissues: endothelial/ MuSCs ratio was 6.2 for nuclei derived from the dissociated cells, and 0.7 for nuclei extracted from intact tissue [22]. This bias is likely due to differences in extraction efficiency of cells from intact and dissociated tissues. Careful histological studies on biopsies combined with flow cytometry methods will help determine the actual population ratios. Multinucleated muscle fibres are another example of how cell morphology can impact the representation of tissue composition. Myofibers are large, elongated multinucleated syncytial cells and by far the main cell type of skeletal muscle (roughly 55\% of all the nuclei of skeletal muscles, based on snRNA-seq) yet are absent in single-cell RNA-seq (scRNA-seq) atlases, as it is not possible to isolate and sequence them using standard protocols. Recently, implementation of snRNA-seq on skeletal muscles has uncovered myonuclear subpopulations and coordinated transcriptional properties of the myonuclei that previously were not accessible for analysis [23-25].

Altogether, these results indicate that snRNA-seq is an advantageous method in preventing dissociation-induced artefacts and providing access to the nuclei of syncytia, whilst still enabling large-scale transcriptional profiling experiments with marginal costs and complexity. Nevertheless, the impossibility to select for cell-surface antigens, the lack of cytoplasmic RNAs and a lower sensitivity that could impede the detection of low expressed genes add some limitations to this method. 


\section{$\underline{\text { In situ cell fixation prior to dissociation }}$}

Aldehydes have been used for more than a century to preserve biological samples through the process of fixation [26]. Fixation preserves organic samples and arrests any biological function, including transcription, by cross-linking the macromolecules [27]. Paraformaldehyde (PFA) has been shown to preserve RNA integrity and composition, notably for RNA-seq [11], [28]. Independent studies employed PFA fixation to preserve the nascent transcriptome of isolated cells and abrogate the impact of the dissociation procedure $[11,12]$. Cells were fixed within the tissue of interest prior to dissociation with a low-concentration of $0.5 \%$ PFA, in a process that we termed in Situ Fixation, or $i \mathrm{SiFi}$ for short [11]. Using bulk RNA-seq on iSiFi-treated skeletal MuSCs we made the unexpected observation that close to half of the transcriptome was dysregulated during isolation, with thousands of both upregulated and downregulated transcripts. Importantly, iSiFi efficiently preserved cells' transcriptome and prevented the induction of early-response genes. These results were later confirmed at the protein level and were expanded to describe specific biological functions, as discussed later [22], [29, 30]. Recently, the fixative glyoxal has shown some superiority in term of RNA-quality for transcriptional studies after cell sorting [31]. Single-cell studies, methanol has also been used as a fixative agent to preserve the transcriptome of mouse kidney cells [21] and mouse inner ear cells, successfully allowing the identification of rare cell types, notably spindle and root cells [32]

The use of fixation prior to dissociation for gene expression preservation is a low-cost procedure, using accessible chemicals with minor modifications to existing protocols. iSiFi-treated cells are further compatible with chromatin immunoprecipitation (ChIP) for analysis of histone $\mathrm{H} 3$ modifications and specific transcription factors, and DNA methylation analysis by reduced representation bisulfite sequencing (RRBS) [11], [33]. Of note, however, to date PFA-fixed cells are not compatible with high throughput single-cell platforms, such 10XGenomics, while methanol-fixed seems to be for some cell systems [34].

\section{Cell dissociation with cold active proteases}

An alternate approach to mitigate dissociation-induced modifications is by performing tissue dissociation at low temperatures using cold-active enzymes to decrease cellular activity and arrest transcription. An elegant study by Adam et al. confirmed this notion by using a psychrophilic protease isolated from the Himalayan glacier bacterium Bacillus licheniformis, to digest developing mouse kidney tissue for $15 \mathrm{~min}$ at $6{ }^{\circ} \mathrm{C}$ with the serine protease subtilisin A [14]. Since then, several studies reported similar findings using cryophilic proteases. In mouse ovarian and breast tumours, a core set of 512 genes induced by standard procedures was identified using a similar cold digestion protocol [35]. Furthermore, in adult murine hippocampus, low temperature mechanical dissociation at $4^{\circ} \mathrm{C}$ was also shown to prevent the induction of stress response genes [36]. Comparable findings were attained in the murine kidney [21], retina [37] and inner-ear [38].

To date, our understanding of the cold-shock response in eukaryotes is limited. Several studies have demonstrated that exposing mammalian cells to sub-physiological temperatures invokes a coordinated cellular response involving induction of cold-shock proteins and modulation of translation, metabolism, cell cycle and the cell cytoskeleton [39]. Therefore, although cold digestion prevents, to a large extent, the dissociation-induced artefacts, it is still possible to alter in some way the in vivo molecular profile of the cells, for example, cryopreserved cells experiment some induction of earlyresponse genes [40]. Of note, cells isolated using standard procedures also commonly experience cold-stress during several steps of the protocol, such as centrifugation and transfer of cells, as these steps are performed at $4{ }^{\circ} \mathrm{C}$ and on ice, respectively. Cold digestion is one of the few procedures described here that keeps the cells alive, which can be a critical asset for downstream applications. 
Furthermore, cold digestion is compatible with standard RNA extraction and sequencing kits including 10xGenomics, without protocol specific adaptations. The use of cold active proteases represents a promising avenue toward stress-free transcriptional studies and determination of the range of tissues in which it could be effectively applied to, would be of great interest.

\section{$\underline{\text { Inhibitors during the cell dissociation }}$}

Similar to cold digestion, slowing down or blocking the transcriptional machinery with inhibitory compounds has the potential to reduce experimentally introduced changes. Digestion of mouse skeletal muscle in the presence of $\alpha$-amanitin, a selective inhibitor of RNA polymerase II and III, was successfully associated with a lower dissociation-induced signature [12] and in the mouse brain, the potent transcriptional inhibitor actinomycin $\mathrm{D}$ elicited similar results $[38,39]$. In another study, a cocktail of chemicals aimed to block calcium entry, neuronal activity, transcription and translation also prevented dissociation-induced genes expression [43]. However, these methods target modifications driven by neo-transcription, but miss other alterations such as gene repression, posttranscriptional modifications and more. Furthermore, it is difficult to ensure total and homogeneous diffusion of the chemical inhibitors in tissues, as well as their specificity. In this regard, we recognize the potential to mitigate the dissociation-induced signature by these methods, but we believe that more studies are necessary to assess the activity of alternative RNA regulation mechanisms under transcriptional inhibition mechanisms, and the extent to which the generated atlases will be free from artefacts.

\section{Cell-type specific nascent RNA tagging}

Conditional labelling of nascent RNA in specific cell types is a sophisticated tool to study gene expression in vivo [41, 42]. Such methods can be applied to avoid dissociation-induced artefacts when the induction of RNA tagging precedes the cell-isolation procedure. For example, Thiouracil (TU)tagging induced with the MuSC-specific Pax7-CreERT2 recombinase driver, permitted the isolation and sequencing of the quiescent transcriptome, free from artefacts [12]. Ribosomal-tagging was similarly used in mouse microglia [46] and inner-ear [38], [47] to label RNA prior to cell dissociation and thus dampen the effect of the procedure. These methods are precise and elegant, allowing the spatial and temporal control of RNA labelling in vivo. However, due to the complexity of these procedures they are difficult to implement in diverse biological systems.

\section{$\underline{P o s t}$ hoc bioinformatics correction of dissociation-induced modifications}

Considering the intellectual and financial investments that are injected into ongoing and published transcriptomic studies, it would be widely useful if the dissociation-induced modifications could be retrospectively attenuated or completely removed from existing datasets. Indeed, it is tempting to postulate that given a sufficient data volume, reducing these alterations computationally would be possible. Most recent studies have relied on complex machine learning algorithms, both unsupervised and supervised mutual nearest neighbours detection, to untangle and correct biological variation from technical noise $[45,46]$. These tools perform under the assumption that biological variability (e.g., the difference between cell types) is much greater than batch effect-induced variability (e.g., technical noise due to the preparation method).

Several groups took the challenge of correcting for dissociation-induced artefacts in silico. Some studies removed cells expressing high stress signatures from downstream analyses; this accounted for approximately $10 \%$ of the cells in a study done on human prostate cells [50]. Other studies removed only stress genes [48-53], both stress genes and stressed cells [57], or regressed out dissociationinduced genes as a confounding variable from their analyses $[55,56]$. Most of the studies cited above 
cleaned their datasets by excluding a limited set of less than 200 dissociation-induced genes, based on an earlier study on MuSCs [13], whilst the literature suggests that $10-50 \%$ of the transcriptome becomes differentially expressed by dissociation [22]. Ironically, the proportion of a cell's transcriptome altered by dissociation is so large that trying to regress the stress artefact out of it might alter it even further, creating artificial reference maps, distant from the in vivo profile.

Recent systematic and temporal analysis on dissociation-induced stress [22] suggests that given enough time every cell gets altered by the dissociation procedures, and that each cell-type reacts idiosyncratically and dynamically to it. Moreover, most batch effect corrections assume orthogonality in the biological sub-space, i.e., that all cells within the same experiment undergo the same batch effect. For these reasons, it appears technically challenging to infer for each cell-type, in each biological setup, the optimal gene adjustment required to remove the specific stress stimuli along a dynamic stimulation trajectory.

In our opinion, corrections a posteriori for noise factor should only be considered as a last resort since specialized tools now exist, presented in this review, to prevent it. Nevertheless, in silico corrections can be a helpful tool to improve existing datasets if a cell-type specific, biologically adapted set of tools is available for correction.

\section{Spatially resolved transcriptomics}

Despite not having systematically studied dissociation-induced genes, it is explicit that spatial transcriptomics methods performed on tissue sections [57-60] will preserve the cells from dissociation-induced modifications. These methods, comprehensively reviewed elsewhere [64], were designed to perform high-throughput transcriptomic profiling of cells in situ, therefore, conserving precious topological information about tissue architecture, cell interactions and transcript localization without requiring cellular isolation. To a lesser extent, laser-capture microdissection on frozen samples coupled with next generation sequencing is an adapted approach for retrieving transcriptome information linked to an anatomical location, whilst circumventing dissociation-induced artefacts. Examples exist in human and mouse brain microvessels [65], as well as on single neurons by aspirating the cytoplasmic content of a neuron following electrophysiological measurements [66]. To date, these methods do not exhibit the simplicity, scalability, resolution (often not single-cell), sequencing depth, genome coverage and ecosystem of analysis tools necessary to substitute cellisolation based methods. Unquestionably, however, spatial transcriptomics will become the technology of choice for transcriptomic studies, as the breadth of knowledge stemming from the topological information is unmatched.

Collectively, most of the methods described in this review are easily implemented. Nevertheless, a simple guideline for stress-free preparations is described in Box 1.

\section{Case studies: how dissociation-induced artefacts can mask dynamic biological functions}

Comparative studies might be mostly spared interference by dissociation, assuming that the artefacts are equivalent between conditions and hence cancelled out (Figure 1A). In studies aiming to represent the absolute profile of cells, such as in reference atlases, dissociation-induced modifications are readily detected, however, they retain adequate accuracy to yield comprehensive results (Figure 1B). The strongest interference due to dissociation-induced artefacts, in our view, has to do with the interpretation of biological functions in dynamic cellular systems. Many processes take place in a timeframe that is an order of magnitude shorter than tissue dissociation itself, such as signal transduction, transcription factor binding or membrane depolarization. Here, in Box 2 and Box 3, two case studies are described that demonstrate how the methods presented above can be harnessed to 
circumvent dissociation-induced stress and give insight to previously inaccessible biological processes (Figure 1C).

\section{Concluding Remarks and Future Perspectives}

We presented here currently available methods to circumvent dissociation-induced artefacts including cold-dissociation, snRNA-seq, in situ fixation, the use of chemical inhibitors, nascent transcriptome pull-down, bioinformatic post-hoc corrections and spatial transcriptomics. This review aims to provide a roadmap for stress management in high-throughput transcriptional studies. Far from perfect, every experimental intervention brings its own set of trade-offs, artefacts, and complications (see Outstanding Questions). Therefore, a careful analysis of the benefits and risks of each method is necessary for choosing for each project the most adapted procedure, which will yield the most accurate transcriptional maps.

This review serves as a cautionary tale of data interpretation on cells isolated from their in vivo environment. More importantly, it aims to promote the development of more sophisticated methods to accurately capture single-cell states as they exist in their native tissue context and towards the construction of second-generation transcriptional atlases. 


\section{Box 1. A simple guideline for stress-free preparations.}

The future gold-standard for transcriptomic studies will be free of dissociation, in the form of spatial transcriptomics methods. However, to this day, such methods remain out-of-reach for most projects. A simple, practical guideline stemming from our work is to quickly dissociate the tissues, whenever possible. Timecourse experiments in muscle stem cells and systematic analysis of published literature in diverse tissues, indicate that digestion time of $30 \mathrm{~min}$ or less induced only marginal transcriptional alterations [22]. Of note, notwithstanding cell type differences, the time required for FACS seems to be less important as the cell isolation procedure has been reported to have only a minor impact on the transcriptome [67]. The majority of tissues from the Tabula Muris cell atlas [5] escape encounters of dissociation-induced artefacts through this simple route, demonstrating how well-managed, methodological practices can yield great results. When celerity is unattainable, however, efforts must be directed towards preserving cells' in vivo molecular state using the methods described in the present review. Regardless of the experimental strategy, downstream datasets must be systematically evaluated for signs of transcriptional stress, and bioinformatic interventions could be employed to reduce data distortions. 


\section{Box 2. Cell dissociation fires neuronal signals that overlap with activity-dependent transcription.}

A prominent example of interference of data acquisition and experimental methodology comes from neurological studies on activity-dependent transcription. Discovered almost 40 years ago [68], Fos became the prototypical activity-regulated gene. Like the rest of its gene family, Fos becomes activated by calcium signalling, is a reporter of neuronal activity (e.g., membrane depolarization), plays a role in engram formation, synaptic plasticity and long-term adaptation, and is also a dissociation-induced gene [69]. How can one correlate the transcriptional response to each one of these processes given their respective kinetics -from minutes to days- and the distorting impact of dissociation? When B. Lacar and colleagues used standard single-cell RNAseq to evaluate the transcriptome of dissociated hippocampal neurons exposed to an epilepsy-inducing drug (PTZ), they scored, as expected, high levels of Fos. However, they observed that Fos and other activity-dependent genes were also detected in their untreated control group, in direct contrast to in situ hybridization experiments showing virtually no such transcripts in resting hippocampal neurons [10]. Only when the team employed single-nuclei RNAseq were they able to circumvent this caveat and uncover the actual activity-dependent transcriptomic signature in the hippocampal neurons in response to a novel environment. Harnessing this method allowed researchers to generate an accurate snRNA-seq transcriptomic atlas of more than 18,000 neurons, which delineated a continuum of heterogeneous activation states [70]. In a separate study, the rapid transcriptional response of neurons to light stimulation was investigated [43]. However, researchers found that the dissociated neurons from mice that were kept in the dark exhibited a strong activity-dependent profile similar to that of the light-stimulated group, preventing any meaningful interpretation. When the researchers applied a cocktail of chemical inhibitors to dampen cellular activation, a lightstimulated transcriptional program was uncovered together with cellular heterogeneity in response to this stimulation, both between and inside cortical layers [43], further emphasizing the importance of mitigating of dissociation-induced artefacts for the precise study of the biology of neurons. 


\section{Box 3. How skeletal muscle stem cells quiescence was escaping us for years.}

A paradigm of how specialised methods can help us uncover up-to-date inaccessible biological processes and introduce new concepts, stems from studies on quiescence exit of skeletal MuSCs. These cells, like all quiescent stem cells, have the capacity to stay in a reversible $\mathrm{G} 0$-arrested state, whilst retaining the ability to regenerate muscle tissue upon injury [71]. In accordance with the work on neurons presented above, initial transcriptomic studies on freshly isolated MuSCs from resting muscle found strong enrichment of early-response genes and pro-mitotic oncogenes. This conundrum puzzled the field for years and it was hypothesized that skeletal MuSCs were molecularly poised for activation in response to injury [72]. By iSiFi and snRNA-seq studies, it was later established that freshly-isolated MuSCs were in fact prematurely activated upon isolation and transcriptionally estranged from quiescence [11, 12], [22]. Moreover, these and subsequent studies enabled the unveiling of biologically relevant determinants of MuSC activation, including the rapid upregulation of genes encoding glycolytic enzymes at the expense of fattyacid oxidation transporters and enzymes [11], [73], the global increase of transcript splicing via the phosphorylation of DEK [30], and the induction of polyamine biosynthesis pathway via the induction of ornithine decarboxylase $(O d c)$ [22]. These studies defined a new "early activation" state of MuSCs [11, 12], [22], [29, 30] and found that during this step, an essential ERK1/2 primary proliferation signal preceded the initiation of the Notch-regulated myogenic program [22]. By combining ERK1/2 MAPK inhibition with Notch signalling induction, ex vivo skeletal MuSCs were maintained ex vivo in a proto-quiescent state [22], underlining the functional relevance of the improved transcriptome methods presented here. Finally, these results were confirmed through the genetic impairment of dissociation-induced responses in MuSCs by knocking-off the early-response gene Fos, which impaired their regenerative capacity [74]. 


\section{Acknowledgements}

FR laboratory is funded by the Association Française contre les Myopathies (AFM) via TRANSLAMUSCLE (PROJECT 19507 and 22946), Labex REVIVE (ANR-10-LABX-73), Fondation pour la Recherche Médicale (FRM; Grant DEQ20130326526), Agence Nationale pour la Recherche (ANR) grants BMP-MyoStem (ANR-16-CE14-0002-03), MyoStemVasc (ANR-17-CE140018-01), Myolinc (ANR- R17062KK) to FR, and muscleXTRA (ANR -19-CE13-0010-01), MyoFibrosis (ANR-19-CE14-0008-03) and to PM.

\section{Declaration of Interests}

The authors declare no competing interests.

\section{References}

[1] R. Hooke, Micrographia, or, Some physiological descriptions of minute bodies made by magnifying glasses with observations and inquiries thereupon / by R. Hooke. 1665.

[2] T. Schwann, Mikroskopische Untersuchungen über die Uebereinstimmung in der Struktur und dem Wachsthum der Thiere und Pflanzen. 1839.

[3] F. Crick, “Central Dogma of Molecular Biology,” Nature, vol. 227, no. 5258, pp. 561-563, 1970.

[4] F. Edfors et al., "Gene-specific correlation of RNA and protein levels in human cells and tissues," pp. 1-10, 2016.

[5] N. Schaum et al., "Single-cell transcriptomics of 20 mouse organs creates a Tabula Muris," Nature, vol. 562, no. 7727, pp. 367-372, 2018.

[6] X. Han et al., "Mapping the Mouse Cell Atlas by Microwell-Seq," Cell, vol. 172, no. 5, pp. 1091--1107.e17, Feb. 2018.

[7] A. Regev et al., "The human cell atlas," Elife, vol. 6, pp. 1-30, 2017.

[8] E. C. Baechler et al., "Expression levels for many genes in human peripheral blood cells are highly sensitive to ex vivo incubation," Genes Immun., vol. 5, no. 5, pp. 347-353, 2004.

[9] W. C. Hines, Y. Su, I. Kuhn, K. Polyak, and M. J. Bissell, "Sorting Out the FACS: A Devil in the Details," Cell Rep., vol. 6, no. 5, pp. 779-781, Mar. 2014.

[10] B. Lacar et al., "Nuclear RNA-seq of single neurons reveals molecular signatures of activation," Nat. Commun., 2016.

[11] L. Machado et al., "In Situ Fixation Redefines Quiescence and Early Activation of Skeletal Muscle Stem Cells," Cell Rep., vol. 21, no. 7, 2017.

[12] C. T. J. van Velthoven, A. de Morree, I. M. Egner, J. O. Brett, and T. A. Rando, "Transcriptional Profiling of Quiescent Muscle Stem Cells In Vivo," Cell Rep., vol. 21, no. 7, pp. 1994-2004, 2017.

[13] S. C. van den Brink et al., "Single-cell sequencing reveals dissociation-induced gene expression in tissue subpopulations," vol. 14, no. 10, 2017.

[14] M. Adam, A. S. Potter, and S. S. Potter, "Psychrophilic proteases dramatically reduce single 
cell RNA-seq artifacts : A molecular atlas of kidney development," no. August, 2017.

[15] R. V Grindberg et al., "RNA-sequencing from single nuclei," Proc. Natl. Acad. Sci. U. S. A., vol. 110, no. 49, pp. 19802-19807, Dec. 2013.

[16] N. Habib et al., "Div-Seq: Single-nucleus RNA-Seq reveals dynamics of rare adult newborn neurons," Science, vol. 353, no. 6302, pp. 925-928, Aug. 2016.

[17] B. B. Lake et al., "Neuronal subtypes and diversity revealed by single-nucleus RNA sequencing of the human brain," Science, vol. 352, no. 6293, pp. 1586-1590, Jun. 2016.

[18] D. Gaidatzis, L. Burger, M. Florescu, and M. B. Stadler, "Analysis of intronic and exonic reads in RNA-seq data characterizes transcriptional and post-transcriptional regulation," Nat. Biotechnol., vol. 33, p. 722, Jun. 2015.

[19] T. E. Bakken et al., "Single-nucleus and single-cell transcriptomes compared in matched cortical cell types," PLoS One, 2018.

[20] H. Wu, Y. Kirita, E. L. Donnelly, and B. D. Humphreys, “Advantages of Single-Nucleus over Single-Cell RNA Sequencing of Adult Kidney : Rare Cell Types and Novel Cell States Revealed in Fibrosis," pp. 23-32, 2019.

[21] E. Denisenko et al., "Systematic assessment of tissue dissociation and storage biases in singlecell and single-nucleus RNA-seq workflows," Genome Biol., vol. 21, no. 1, p. 130, Jun. 2020.

[22] L. Machado et al., "Tissue damage induces a conserved stress response that initiates quiescent muscle stem cell activation," Cell Stem Cell, Feb. 2021.

[23] M. Kim et al., "Single-nucleus transcriptomics reveals functional compartmentalization in syncytial skeletal muscle cells," Nat. Commun., vol. 11, no. 1, p. 6375, 2020.

[24] M. J. Petrany et al., "Single-nucleus RNA-seq identifies transcriptional heterogeneity in multinucleated skeletal myofibers.," Nat. Commun., vol. 11, no. 1, p. 6374, Dec. 2020.

[25] M. Dos Santos et al., "Single-nucleus RNA-seq and FISH identify coordinated transcriptional activity in mammalian myofibers," Nat. Commun., 2020.

[26] C. H. Fox, F. B. Johnson, J. Whiting, and P. P. Roller, "Formaldehyde fixation.," J. Histochem. Cytochem., vol. 33, no. 8, pp. 845-853, Aug. 1985.

[27] R. Thavarajah, V. K. Mudimbaimannar, J. Elizabeth, U. K. Rao, and K. Ranganathan, "Chemical and physical basics of routine formaldehyde fixation," J. Oral Maxillofac. Pathol., vol. 16, no. 3, pp. 400-405, Sep. 2012.

[28] E. R. Thomsen et al., "Fixed single-cell transcriptomic characterization of human radial glial diversity," Nat Methods, vol. 13, no. 1, pp. 87-93, 2016.

[29] N. Pietrosemoli et al., "Comparison of multiple transcriptomes exposes unified and divergent features of quiescent and activated skeletal muscle stem cells," Skelet. Muscle, vol. 7, no. 1, p. 28, Dec. 2017.

[30] L. Yue, R. Wan, S. Luan, W. Zeng, and T. H. Cheung, "Dek Modulates Global Intron Retention during Muscle Stem Cells Quiescence Exit," Dev. Cell, vol. 53, no. 6, pp. 661676.e6, 2020.

[31] P. Channathodiyil and J. Houseley, "Glyoxal fixation facilitates transcriptome analysis after antigen staining and cell sorting by flow cytometry," PLoS One, vol. 16, no. 1, p. e0240769, Jan. 2021.

[32] S. Gu et al., "Characterization of rare spindle and root cell transcriptional profiles in the stria 
vascularis of the adult mouse cochlea," Sci. Rep., vol. 10, no. 1, p. 18100, 2020.

[33] M. B. Baghdadi et al., "Reciprocal signalling by Notch-Collagen V-CALCR retains muscle stem cells in their niche," Nature, vol. 557, no. 7707, pp. 714-718, 2018.

[34] J. Chen et al., "PBMC fixation and processing for Chromium single-cell RNA sequencing," $J$. Transl. Med., vol. 16, no. 1, p. 198, 2018.

[35] C. H. O'Flanagan et al., "Dissociation of solid tumor tissues with cold active protease for single-cell RNA-seq minimizes conserved collagenase-associated stress responses," Genome Biol., vol. 20, no. 1, p. 210, 2019.

[36] D. Mattei et al., "Enzymatic dissociation induces transcriptional and proteotype bias in brain cell populations," Int. J. Mol. Sci., vol. 21, no. 21, pp. 1-20, 2020.

[37] B. R. Fadl et al., "An optimized protocol for retina single-cell RNA sequencing,” Mol. Vis., vol. 26, pp. 705-717, Oct. 2020.

[38] R. Hertzano, K. Gwilliam, K. Rose, B. Milon, and M. S. Matern, "Cell Type-Specific Expression Analysis of the Inner Ear: A Technical Report," Laryngoscope, vol. n/a, no. n/a, Jun. 2020.

[39] B. J. Fuller, "Gene expression in response to low temperatures in mammalian cells: a review of current ideas.," Cryo Letters, vol. 24, no. 2, pp. 95-102, 2003.

[40] B. Morsey et al., "Cryopreservation of microglia enables single-cell RNA sequencing with minimal effects on disease-related gene expression patterns," iScience, vol. 24, no. 4, p. $102357,2021$.

[41] Y. E. Wu, L. Pan, Y. Zuo, X. Li, and W. Hong, "Detecting Activated Cell Populations Using Single-Cell RNA-Seq," Neuron, 2017.

[42] H. Van Hove et al., "A single-cell atlas of mouse brain macrophages reveals unique transcriptional identities shaped by ontogeny and tissue environment," Nat. Neurosci., vol. 22, no. 6, pp. 1021-1035, 2019.

[43] S. Hrvatin et al., "Single-cell analysis of experience-dependent transcriptomic states in the mouse visual cortex," Nat. Neurosci., vol. 21, no. 1, pp. 120-129, Jan. 2018.

[44] E. Sanz, L. Yang, T. Su, D. R. Morris, G. S. McKnight, and P. S. Amieux, "Cell-type-specific isolation of ribosome-associated mRNA from complex tissues," Proc. Natl. Acad. Sci., vol. 106, no. 33, pp. 13939 LP - 13944, Aug. 2009.

[45] L. Gay et al., "Mouse TU tagging: a chemical/genetic intersectional method for purifying cell type-specific nascent RNA.," Genes Dev., vol. 27, no. 1, pp. 98-115, Jan. 2013.

[46] Z. Haimon et al., "Re-evaluating microglia expression profiles using RiboTag and cell isolation strategies /631/1647/2017/631/1647/2017/2079 technical-report," Nat. Immunol., vol. 19, no. 6, pp. 636-644, 2018.

[47] E. Sadler et al., "Cell-Specific Transcriptional Responses to Heat Shock in the Mouse Utricle Epithelium ," Frontiers in Cellular Neuroscience , vol. 14. p. 123, 2020.

[48] L. Haghverdi, A. T. L. Lun, M. D. Morgan, and J. C. Marioni, "Batch effects in single-cell RNA-sequencing data are corrected by matching mutual nearest neighbors," Nat. Biotechnol., vol. 36, no. 5, pp. 421-427, Jun. 2018.

[49] Y. Yang, G. Li, H. Qian, K. C. Wilhelmsen, Y. Shen, and Y. Li, "SMNN: batch effect correction for single-cell RNA-seq data via supervised mutual nearest neighbor detection," Brief. Bioinform., Jun. 2020. 
[50] G. H. Henry et al., "A Cellular Anatomy of the Normal Adult Human Prostate and Prostatic Urethra," Cell Rep., vol. 25, no. 12, pp. 3530-3542.e5, 2018.

[51] D. B. Joseph et al., "Urethral luminal epithelia are castration-insensitive cells of the proximal prostate," Prostate, vol. 80, no. 11, pp. 872-884, 2020.

[52] E. Ydens et al., "Profiling peripheral nerve macrophages reveals two macrophage subsets with distinct localization, transcriptome and response to injury," Nat. Neurosci., vol. 23, no. 5, pp. 676-689, 2020.

[53] E. Giacomelli et al., "Human-iPSC-Derived Cardiac Stromal Cells Enhance Maturation in 3D Cardiac Microtissues and Reveal Non-cardiomyocyte Contributions to Heart Disease," Cell Stem Cell, vol. 26, no. 6, pp. 862-879.e11, 2020.

[54] J.-J. Chung et al., "Single-Cell Transcriptome Profiling of the Kidney Glomerulus Identifies Key Cell Types and Reactions to Injury," J. Am. Soc. Nephrol., vol. 31, no. 10, pp. 2341 LP 2354, Oct. 2020.

[55] S. Hrvatin et al., "Neurons that regulate mouse torpor," Nature, vol. 583, no. 7814, pp. 115$121,2020$.

[56] L. Yang et al., "A Human Pluripotent Stem Cell-based Platform to Study SARS-CoV-2 Tropism and Model Virus Infection in Human Cells and Organoids," Cell Stem Cell, vol. 27, no. 1, pp. 125-136.e7, 2020.

[57] R. A. Romanov et al., "Molecular design of hypothalamus development," Nature, vol. 582, no. 7811, pp. 246-252, 2020.

[58] R. Massoni-Badosa et al., "Sampling time-dependent artifacts in single-cell genomics studies," Genome Biol., vol. 21, no. 1, pp. 1-16, 2020.

[59] H. Xi et al., "A Human Skeletal Muscle Atlas Identifies the Trajectories of Stem and Progenitor Cells across Development and from Human Pluripotent Stem Cells," Cell Stem Cell, vol. 27, no. 1, pp. 158-176.e10, 2020.

[60] J. H. Lee et al., "Fluorescent in situ sequencing (FISSEQ) of RNA for gene expression profiling in intact cells and tissues," Nat. Protoc., vol. 10, p. 442, Feb. 2015.

[61] G. Wang, J. R. Moffitt, and X. Zhuang, "Multiplexed imaging of high-density libraries of RNAs with MERFISH and expansion microscopy," Sci. Rep., 2018.

[62] S. G. Rodriques et al., "Slide-seq: A scalable technology for measuring genome-wide expression at high spatial resolution," Science (80-. )., vol. 363, no. 6434, pp. 1463 LP - 1467, Mar. 2019.

[63] C. H. L. Eng et al., "Transcriptome-scale super-resolved imaging in tissues by RNA seqFISH+," Nature, 2019.

[64] J. Liao, X. Lu, X. Shao, L. Zhu, and X. Fan, “Uncovering an Organ's Molecular Architecture at Single-Cell Resolution by Spatially Resolved Transcriptomics.," Trends Biotechnol., vol. 39, no. 1, pp. 43-58, Jan. 2021.

[65] H. W. Song, K. L. Foreman, B. D. Gastfriend, J. S. Kuo, S. P. Palecek, and E. V Shusta, "Transcriptomic comparison of human and mouse brain microvessels," Sci. Rep., vol. 10, no. 1, p. 12358, 2020.

[66] S. Mohammad et al., "Kcnn2 blockade reverses learning deficits in a mouse model of fetal alcohol spectrum disorders," Nat. Neurosci., vol. 23, no. 4, pp. 533-543, 2020.

[67] G. M. Richardson, J. Lannigan, and I. G. Macara, "Does FACS perturb gene expression?," 
Cytometry. A, vol. 87, no. 2, pp. 166-175, Feb. 2015.

[68] M. E. Greenberg and E. B. Ziff, "Stimulation of 3 T3 cells induces transcription of the c-fos proto-oncogene," Nature, vol. 311, no. 5985, pp. 433-438, 1984.

[69] E. L. Yap and M. E. Greenberg, "Activity-Regulated Transcription: Bridging the Gap between Neural Activity and Behavior," Neuron, vol. 100, no. 2, pp. 330-348, 2018.

[70] P. Hu, E. Fabyanic, D. Y. Kwon, S. Tang, Z. Zhou, and H. Wu, "Dissecting Cell-Type Composition and Activity-Dependent Transcriptional State in Mammalian Brains by Massively Parallel Single-Nucleus RNA-Seq," Mol. Cell, vol. 68, no. 5, pp. 1006-1015.e7, Dec. 2017.

[71] M. B. Baghdadi and S. Tajbakhsh, "Regulation and phylogeny of skeletal muscle regeneration,” Dev. Biol., vol. 433, no. 2, pp. 200-209, 2018.

[72] L. Liu et al., "Chromatin Modifications as Determinants of Muscle Stem Cell Quiescence and Chronological Aging," Cell Rep., 2013.

[73] J. G. Ryall and G. S. Lynch, "The molecular signature of muscle stem cells is driven by nutrient availability and innate cell metabolism.," Curr. Opin. Clin. Nutr. Metab. Care, vol. 21, no. 4, pp. 240-245, Jul. 2018.

[74] A. E. Almada et al., "FOS licenses early events in stem cell activation driving skeletal muscle regeneration," Cell Rep., vol. 34, no. 4, p. 108656, 2021. 
Table 1. Summary of available methods for mitigating cell dissociation-induced modifications of the transcriptome.

\begin{tabular}{|c|c|c|c|c|c|c|c|}
\hline $\begin{array}{c}\text { high } \\
\text { medium } \\
\text { not } \\
\text { applicable }\end{array}$ & $\begin{array}{c}\text { Cold } \\
\text { digestion }\end{array}$ & Fixation & $\begin{array}{l}\text { Single-nucleus } \\
\text { RNA-seq }\end{array}$ & Inhibitors & $\begin{array}{l}\text { RNA tagging } \\
R_{8}\end{array}$ & $\begin{array}{c}\text { Spatial } \\
\text { transcriptomics }\end{array}$ & Bioinformatics \\
\hline \multicolumn{8}{|l|}{ Accuracy } \\
\hline \multicolumn{8}{|l|}{ Affordability } \\
\hline \multicolumn{8}{|l|}{ Accessibility } \\
\hline \multicolumn{8}{|l|}{ Cell viability } \\
\hline \multicolumn{8}{|l|}{ RNA yield } \\
\hline \multicolumn{8}{|l|}{ Cell number } \\
\hline Strength & $\begin{array}{l}\text { Preserves cells } \\
\text { Reversible }\end{array}$ & $\begin{array}{c}\text { Low-cost } \\
\text { and simple }\end{array}$ & $\begin{array}{c}\text { Low-cost } \\
\text { and simple }\end{array}$ & Preserves cells & $\begin{array}{c}\text { Nascent } \\
\text { transcription } \\
\text { s-/t- control } \\
\end{array}$ & $\begin{array}{c}\text { RNA } \\
\text { topography }\end{array}$ & $\begin{array}{l}\text { Post-hoc } \\
\text { correction }\end{array}$ \\
\hline Weakness & $\begin{array}{l}\text { Potential impact } \\
\text { on transcriptome }\end{array}$ & $\begin{array}{l}\text { Not compatible } \\
\text { with 10x (PFA) }\end{array}$ & $\begin{array}{c}\text { Nuclear } \\
\text { transcriptome }\end{array}$ & $\begin{array}{l}\text { Penetrance } \\
\text { Specificity }\end{array}$ & $\begin{array}{l}\text { Transgenics } \\
\text { limitations }\end{array}$ & $\begin{array}{l}\text { Equipment } \\
\text { Coverage }\end{array}$ & $\begin{array}{c}\text { Data } \\
\text { distortion }\end{array}$ \\
\hline
\end{tabular}


Figure 1. Impact of cell dissociation-induced modifications on single-cell studies.

A State A State B State B

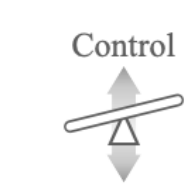

Experimental

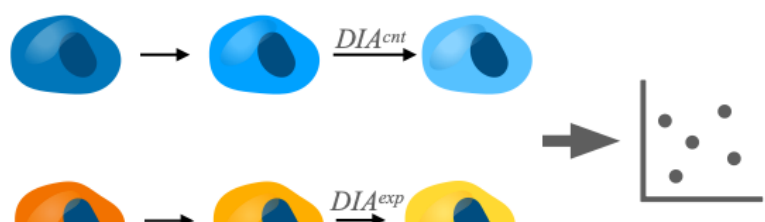

B

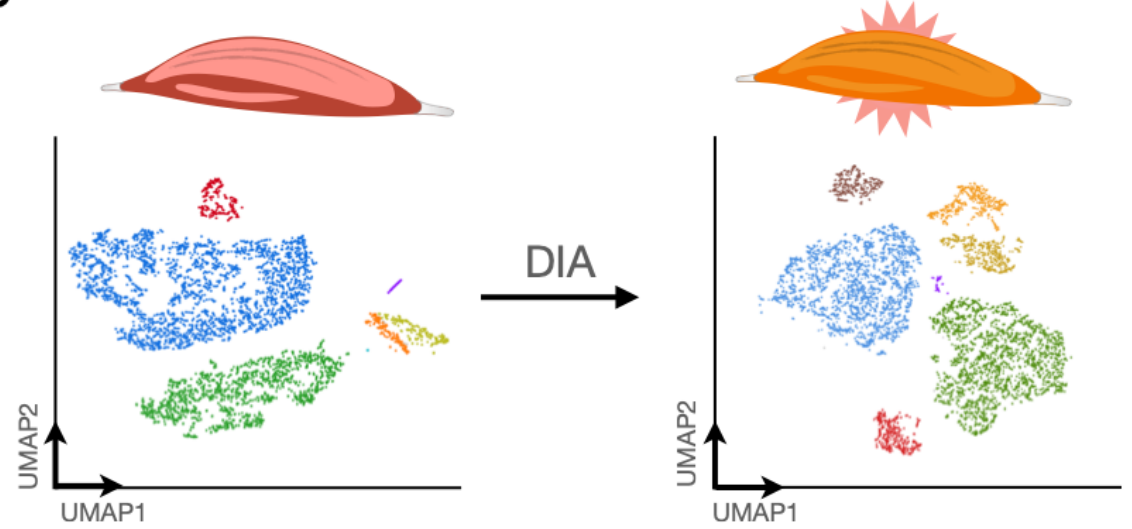

C

Resting neuron

Adaptive behaviour, learning

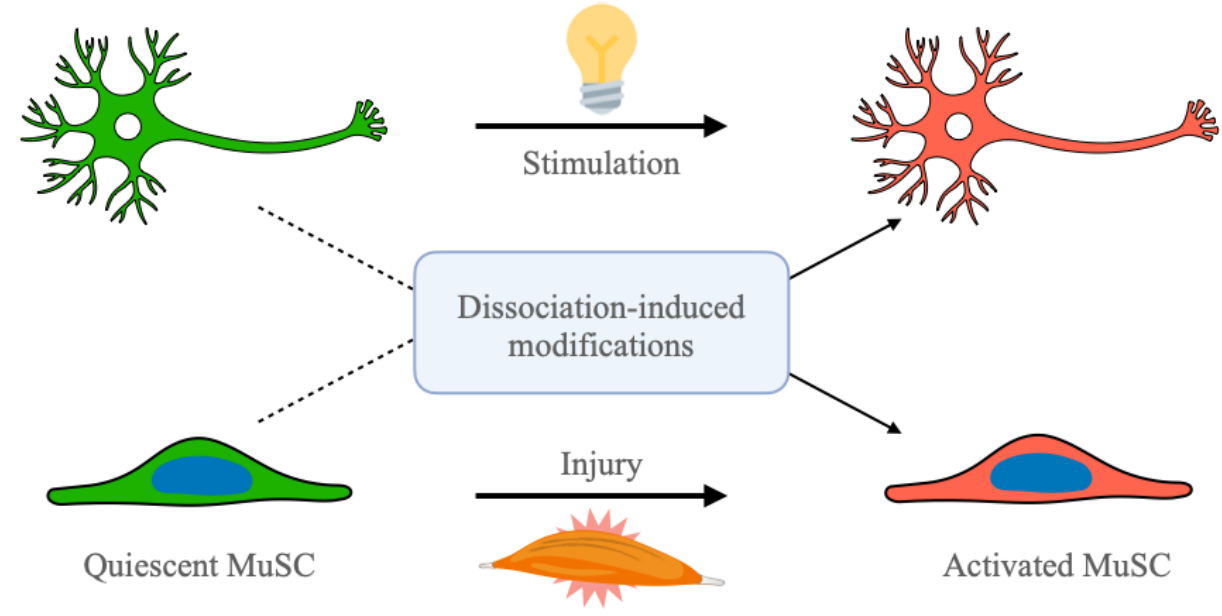

